\title{
LOCAL SUPPLY CHAINS: THE DISASTER MANAGEMENT PERSPECTIVE
}

\author{
KYLE B. PFEIFFER, CARMELLA BURDI \& SCOTT SCHLUETER \\ Risk and Infrastructure Sciences Center, Argonne National Laboratory, United States of America.
}

\begin{abstract}
Situational awareness of the operational status of specific, critical supply and demand nodes following a major disaster may inform response and recovery activities based on the ability of an infrastructure asset or system to support core facility operations. Near-real-time analysis of infrastructure dependency information is a computationally intensive process that has generally been observed informally by public safety officials. While system-level information may be desired, it has been beyond the capabilities of most local public safety and emergency management agencies. To address this problem, a Grassroots Infrastructure Dependency Model (GRID-M) was developed to enable near-real-time analysis of physical infrastructure dependencies of specific supply and demand nodes within four lifeline sectors: electricity, natural gas, water, and wastewater. The operational status of each node can be characterized as operational, partially operational, or not operational. These statuses are obtained by matching real-time outage or disruption data from utility providers with predetermined specific coping strategies based on a preincident limited infrastructure survey for specific assets within a network. This information can also be paired with a limited damage assessment to provide awareness of the accessibility to, and physical state of, each node within supply chains of interest. GRID-M displays all outputs within a Geographic Information Systems environment with additional prepopulated layers such as real-time traffic and demographic information of the affected communities. As such, GRID-M may be used following a major disaster to support the identification of priority response and recovery objectives based on the disruptions of critical local supply chains and their relationship with affected communities.

Keywords: critical infrastructure, dependency, disaster, emergency management, preparedness, resilience, supply chain.
\end{abstract}

\section{INTRODUCTION}

In the context of disaster management, supply chains may be defined as activities associated with the flow and transformation of goods from raw materials to end-users. [1] While other definitions may exist, they are often influenced by industry- or sector-specific attributes that mire the concept for public safety officials. As indicated by Fig. 1, the supply chain concept for this research is rather effort simplistic: materials flow from suppliers to manufacturers who transform the raw materials into finished products. These products are then moved to distributors and eventually received by consumers. This representation of the logistics workflow is useful to scale often complex processes to a manageable paradigm through which local officials can interpret relationships and ultimately affect change regarding the resilience posture of the supply chain.

Managing risk, even for a simplistic supply chain, has become a professional responsibility. In the United States, both small and large private sector organizations employ logisticians, continuity experts, and risk analysts to help identify and mitigate risks to their enterprise. Their focus can be everything from coordination of supply and demand to minimizing disruption of normal activities. For the purpose of this research, disruption risks are examined, as they are more likely to intersect with the roles and responsibilities of public safety officials. [2] Furthermore, those in the public sector may be able to effect the most change around them. Disruption risks can be described in four broad categories: operational contingencies, natural hazards, terrorism, and political instability. Operational contingencies are systematic failures, 


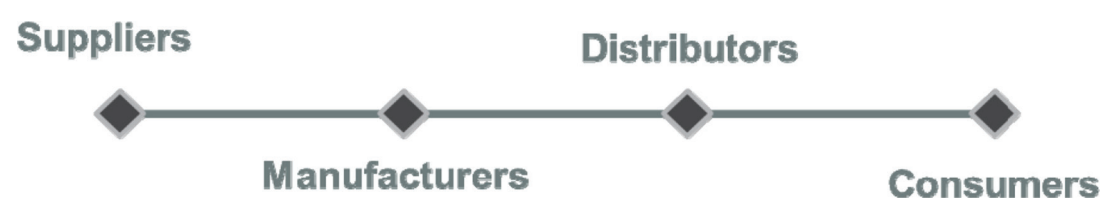

Figure 1: Supply Chain Concept.

such as the August 2003 Northeastern United States grid blackout, which caused curtailment of operations for many supply and demand nodes. [2, 3]. Natural hazards are events such as hurricanes, wildfires, earthquakes, or flooding which can disrupt critical components of a supply chain - such as the fuel shortages in the New York-New Jersey area following Superstorm Sandy in 2012. [4] Terrorism, the next category of disruption risks, may include indiscriminate destruction of critical supply chain components or targeted sabotage of known critical assets and systems. [3] Lastly, political instability may disrupt supply chains, such as oil production curtailment in Iraq and Syria, given the regional strife since 2012.

In the United States, operational contingencies and natural hazards are among the most likely disruption risks to significantly affect supply chains that are critical to communities following a disaster. The effects of these disruptions may directly impact the ability of a community to obtain food, fuel, prescription medication, or other critical goods and services in the minutes, days, or weeks following a disaster. Understanding the behavior of these supply chains, particularly in near real-time after a disaster, may help officials to make more informed decisions regarding response and recovery activities.

\section{FRAMING LOCAL SUPPLY CHAIN RESILIENCE ACTIVITIES}

Public safety officials are often at a disadvantage as compared to their private sector partners when considering risks to their supply chains of concern. First and foremost, most public safety organizations, such as emergency management agencies, have often not identified specific supply chains that provide critical goods and services to their communities nor do they have a precise understanding of the cascading effects of a degradation to one or more of these critical supply chains. This is often due to a combination of a lack of staffing, mission assignment or tasking, and internal expertise. Furthermore, the characteristics of many supply chains make it difficult for public safety officials, particularly at the local level, to effect change; or even build awareness of their risk landscape. This is a result of the global interdependence of supply chains, the untold number of potential disruptors on the worldwide scale, and the lack of public control or influence of private sector logistics (particularly in the United States). These factors make actions led by public sector entities more difficult.

While public safety officials, particularly at the local level, may be hamstrung by these impediments they can still gain awareness of their local supply chain risk landscape and even build near real-time situational awareness of the behavior of their supply chains of concern before, during, and after a disaster. This can be accomplished by scaling the focus area to the relationship between local and nearby distributors, supply nodes (e.g. a grocery store or pharmacy), disaster commodity stockpiles and points-of-distribution, and the affected community. Once the boundary conditions of the supply chains of interest are set, public safety officials can execute a short research and planning project to gain understanding of the likely disruption risks to the Critical Infrastructure (CI) needed to support a given supply chain.

CI consists of systems and assets, whether physical of virtual, that if disrupted, degraded, or destroyed would have a serious impact on the health, safety, security, and well-being 


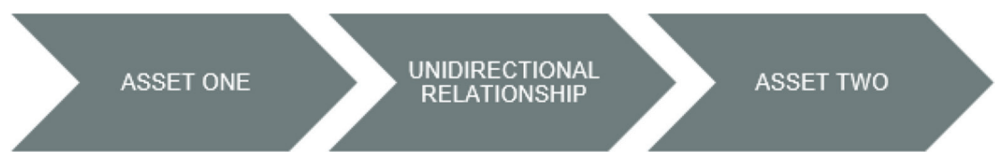

Figure 2: Infrastructure Dependency Concept as Described by Rinaldi et al.

of society. [5, 6] Rinaldi et al. defined a critical infrastructure dependency as a 'linkage or connection between two infrastructures, by which the state of one infrastructure influences or is reliant upon the state of the other'. [7] For example, a cold storage warehouse may be dependent upon an external source of electric power for refrigeration. A disruption to primary electric service, in the absence of a backup generator or battery, may degrade critical operations at the warehouse. In this example, a physical dependency has caused the notional disruption of operations at a cold storage warehouse. This concept is illustrated in Fig. 2.

\section{GRASSROOTS INFRASTRUCTURE DEPENDENCY MODEL}

GRID-M was developed within an Esri environment including ArcGIS for Desktop, ArcGIS for Server, ArcGIS for Portal and/or ArcGIS Online for Organizations (AGOL). GRID-M is located within Esri's model builder application. It is published as a geoprocessing (GP) service to an ArcGIS server in order to be accessible as a defined workflow within a web-based mapping application. This cloud-based access prevents the need for all users to be GIS experts and instead allows for easy access by public safety officials.

The model creation occurs within the Esri ArcGIS Desktop environment. The model builder is 'a flowchart schematic that guides the user to visually lay out the task'. [8] Any ArcGIS for Desktop tools, as well as customized python scripts, are accessible through the model builder and can be customized into a workflow.

The overall model is based on several assumptions regarding the likely supply chains of concern and level of sophistication that local public safety officials have within the logistics domain. First, it is assumed that local public safety officials may cite food, water, fuel, and pharmaceutical supplies among the most critical commodity needs following a major disaster. Furthermore, it is assumed that planners have broad awareness of some of the disruption risks to their local supply chains - particularly in the context of major disasters. While a risk-basis for this framework, combined with a stronger methodology to prioritize essential goods is needed, that was not the focus of this research effort. With time and resources, it is assumed that most planners can make these basic risk-informed judgments to identify their essential supply chains and, in partnership with their private sector partners, identify the likely disruptors in the context of operationally contingent and natural hazard risks. Once that is completed, the initial dataset for the GRID-M would be available.

This initial data set should include a library of georeferenced observation points for each critical supply and demand node and denote the relationship between them (e.g. which distribution warehouses serve which grocery stores). Once this basic dataset is established within the GRID-M, a series of short infrastructure surveys may be completed. These infrastructure surveys are meant to identify reliance on a specific lifeline infrastructure - to include the ability of an entity to cope with a degradation or disruption of service based on coping and continuity strategies. For instance, a grocery store may have a backup generator that can support full operations for a 24-hour period. However, without resupplying on-site fuel, this node will become classified as 'not operational'. An example of the infrastructure survey 
question sets can be found in Fig. 3. Of note, similar question sets are available to assess the dependency of an asset based on reliance on water, wastewater, and natural gas.

These limited infrastructure surveys must be completed for each node. In many instances, corporations will have similar coping strategies (e.g. a generator) so the data entry may be somewhat streamlined. Of note, research is underway to allow for validation or suggested survey data for common supply chains and nodes rather than force public safety officials to conduct extensive preliminary research. However, that is not available at this time.

Once the limited infrastructure surveys are completed they should be validated by the respective private sector entities and then housed in the native GRID-M database.

The next step in the planning process is for public safety officials to establish informal infrastructure reporting requirements with their utility owners and operators. For instance, an emergency management agency may request that the electric utility provider(s) for their community provide outage data when a certain threshold is met. These reporting thresholds may be based on the percentage of a population affected by an outage or when certain critical geographic areas are without power. Other thresholds may be established based on the information needs of the emergency management entity for each lifeline utility. To be used within the GRID-M, the outage data may be provided in several formats, including.CSV, KML, and. SHP files. Outage areas may also be inserted as polygons using the Esri drawing tool.

Once data is received from a utility provider, it may be imported into the GRID-M. This process usually only takes a few seconds if the data is well structured and in a readable format.

Once imported, an analyst will be able to see how supply and demand nodes are affected within the outage area. The results are generated within the GRID-M by comparing the coping strategies defined within the limited infrastructure surveys and generating results

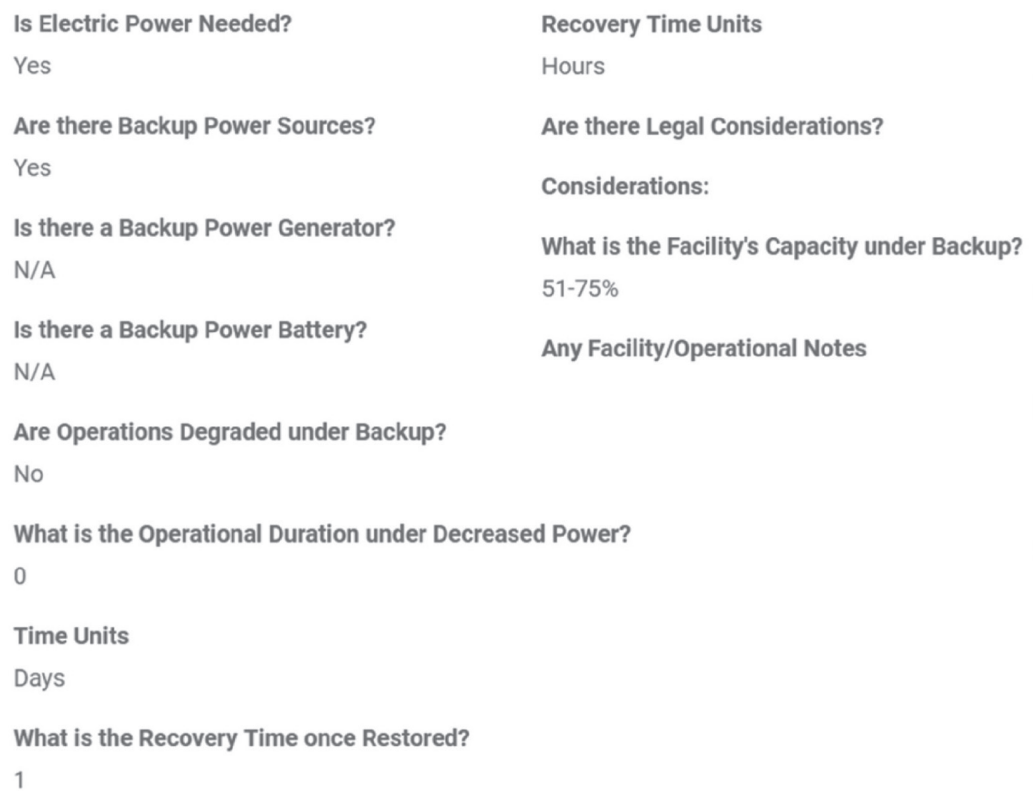


regarding the operational status of each node based on the disruption areas (represented as polygons). The resulting output of the model builder is published as a GP service to an ArcGIS Server. That GP service is available to users of either a Portal or an AGOL account. The creation of a web application serves as a container or UI for the GP service. Configurable widgets house the GP service and provide a direct link for users of the web application to run the GRID-M without the need for an ArcGIS desktop environment.

The use of GRID-M within a cloud-based environment requires Internet Information Services (IIS) to store or host web applications and node.js as a runtime environment.

Figure 4 provides a notional representation of the near-real-time operational status of grocery stores in coastal Maine in the United States based on a disruption of electric service. The white diamonds are fully operational grocery stores, the gray diamonds are partially operational, and the black diamond signifies that the node is non-operational. The squares with bullseyes represent distribution centers. The same color logic applies in this gray scale example.

In another notional example, Fig. 5 depicts a disruption to the regional pharmaceutical supply chain in coastal Maine based on an electric outage.

Of note, in the web application version of GRID-M, the operational status of supply and demand nodes is displayed in a choropleth fashion using a stoplight color scheme.

GRID-M also makes use of the Esri collector application to support limited damage assessments for nodes affected by disruption risks. Using the standard damage assessment form issued by the Federal Emergency Management Agency, survey and assessment teams can report information to Emergency Operations Center staff regarding the physical state of a node. This reporting can be done through a mobile application as well as the desktop and web applications. GRID-M can also support integration with real-time traffic reporting services, such as Waze ${ }^{\mathrm{TM}}$. Therefore, by using this model, public safety officials can receive

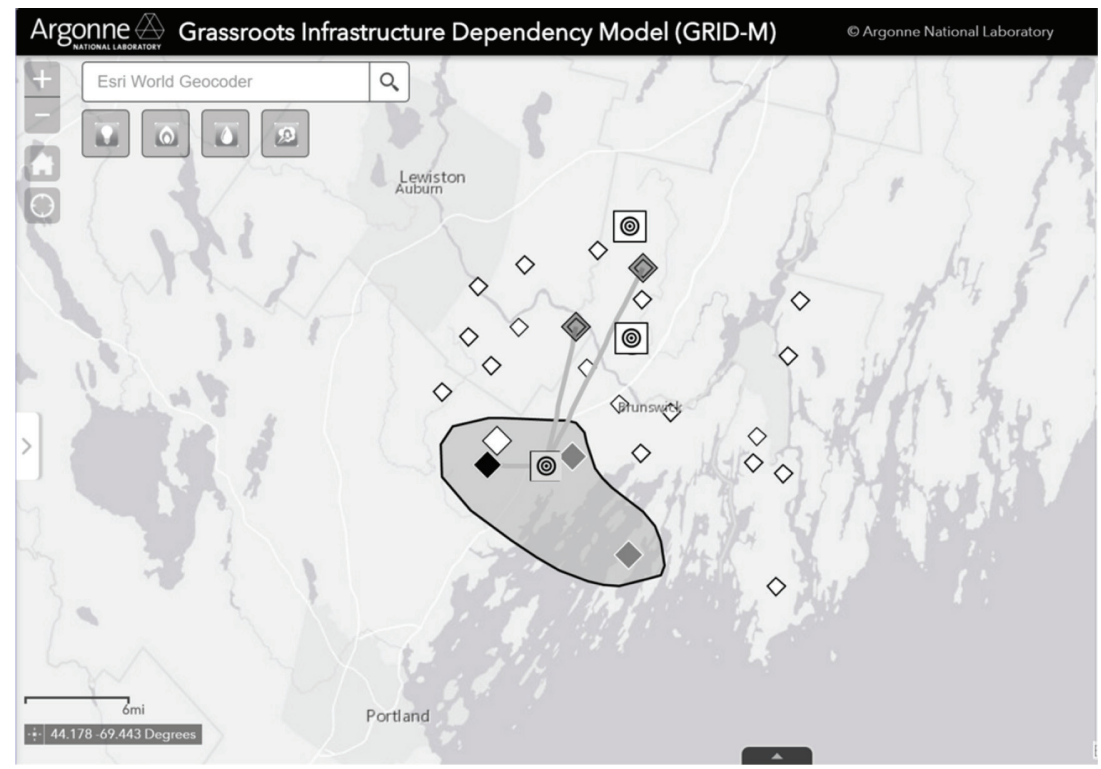

Figure 4: GRID-M Representation of a Notional Disruption to a Local Grocery Supply Chain in Coastal Maine. 


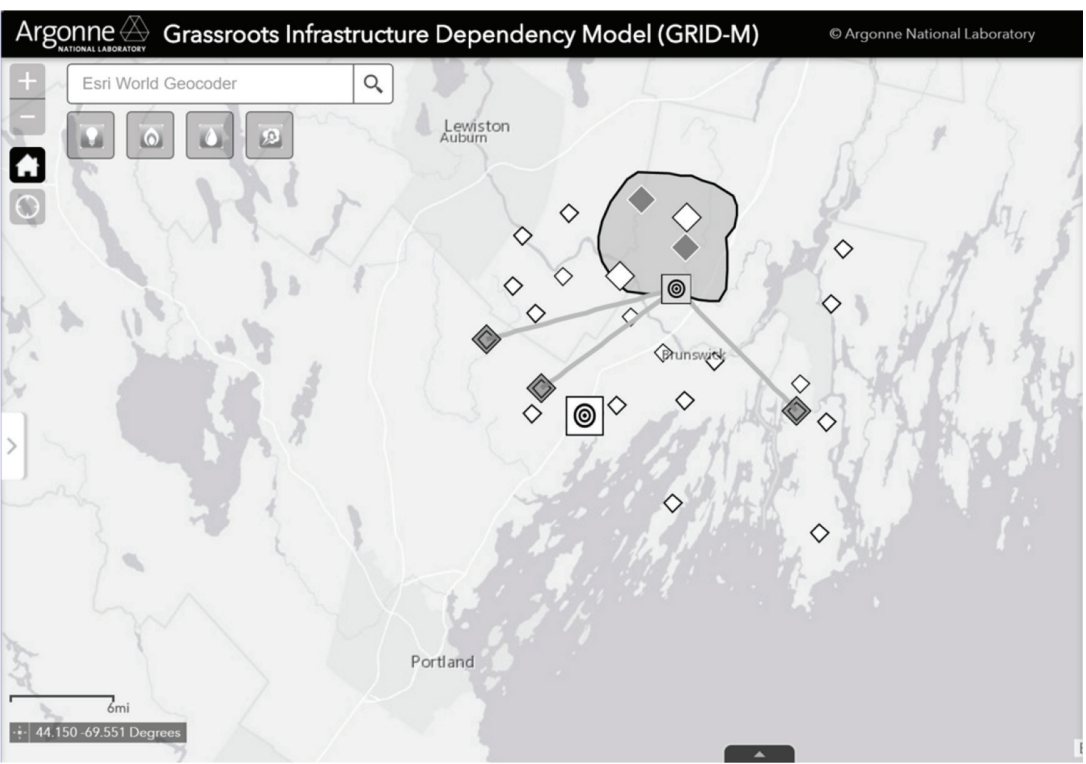

Figure 5: GRID-M Representation of a Notional Disruption to a Local Pharmaceutical Supply Chain in Costal Maine.

moderately vetted information regarding the operational status of an infrastructure based on disruption to primary utility service, its organic coping mechanisms, the physical state of the node, and egress based on surface transportation infrastructure.

\section{CONCLUSIONS}

GRID-M can help public safety officials to make better approximations about disruptions of concern to their supply chains by using preincident survey data regarding the reliance of a node on electric, water, wastewater, and natural gas and its operational capacity and duration following a disruption. GRID-M can also help public safety officials to gain situational awareness on the physical state of a node by using the damage assessment application. This information can support the identification of priority infrastructure for use in planning and exercise simulations as well as real-world situational awareness of the operational status of preidentified supply and demand nodes. It can also feed the incident action planning process, help to prioritize restoration activities, identify suitable shelter sites and points-of-distribution, and even provide quasi-predictive analysis of the local supply chains based on planned restoration activities. While this model has a barrier to entry in the form of collecting data from key private sector partners in a community prior to a disaster, it represents a new paradigm in building a more informed common operating picture that allows for approximations of the behavior of networked assets using vetted data.

The model will be deployed to several cities within the United States by mid-2017.

\section{REFERENCES}

[1] Gaspar, J., Kolari, J., Hise, R., Bierman, L. \& Smith, L.M., Introduction to Global Business: Understanding the International Environment \& Global Business Functions, Cengage Learning: Boston, p. 329, 2017. 
[2] Vakharia, A.J. \& Yenipazarli, A., Managing supply chain disruptions. Foundations and Trends in Technology, Information and Operations Management, 2(4), pp. 245-255, 2009.

[3] Kleindorfer, P.R. \& Saad, G.H., Managing disruption risks in supply chains. Production and Operations Management, 14(1), pp. 53-68, 2005. https://doi.org/10.1111/j.1937-5956.2005.tb00009.x

[4] Hurricane Sandy After-Action Report, United States Department of Homeland Security, Federal Emergency Management Agency, available at https://www.fema.gov/medialibrary-data/20130726-1923-25045-7442/sandy_fema_aar.pdf (accessed 30 January 2017)

[5] National Infrastructure Protection Plan 2013: Partnering for Critical Infrastructure Security and Resilience, United States Department of Homeland Security, available at https://www.dhs.gov/publication/nipp-2013-partnering-critical-infrastructure-securityand-resilience(accessed 30 January 2017)

[6] Presidential Policy Directive-Critical Infrastructure Security and Resilience, The White House, available at https://obamawhitehouse.archives.gov/the-press-office/2013/02/12/ presidential-policy-directive-critical-infrastructure-security-and-resil. (accessed 30 January 2017)

[7] Rinaldi, S., Peerenboom, J. \& Kelly, T., Identifying, understanding and analyzing critical infrastructure interdependencies. IEEE Control Systems, 21(6), pp. 11-25, 2001. https://doi.org/10.1109/37.969131

[8] Allen, D.W., Getting to Know ArcGIS Modelbuilder, Esri Press, Redlands, California: 2011. 